\title{
Conditional Rescue of the dunce Learning/Memory and Female Fertility Defects with Drosophila or Rat Transgenes
}

\author{
Brigitte Dauwalder ${ }^{1, a}$ and Ronald L. Davis ${ }^{1,2}$ \\ 'Department of Cell Biology and ${ }^{2}$ Department of Neurology, Baylor College of Medicine Houston, Texas 77030
}

\begin{abstract}
Mutations in the Drosophila dunce gene, the structural gene for a CAMP-specific phosphodiesterase (PDE), disrupt normal learning and memory and lead to female sterility. Our experiments address a long-standing question in the genetic dissection of learning and memory of whether dnc is involved in physiological processes underlying learning. Conditional expression of dunce transgenes in dnc adults shortly before training significantly improves learning over nontransgenic controls. Remarkably, behavioral rescue was also observed after induction of a transgene carrying a rat counterpart of dunce. Induction of the transgenes in adult dnc females confers partial rescue of the female sterility phenotype. These data are consistent with a major physiological requirement for the gene's activity in the learning process and show that a rat counterpart can substitute functionally for the Drosophila gene.
\end{abstract}

[Key words: rescue of dunce phenotypes, Drosophila, classical conditioning, dunce gene, cAMP phosphodiesterase]

Genetic screens for Drosophila strains defective in associative learning have yielded several mutants that are deficient in a variety of associative and nonassociative learning paradigms (Davis, 1993). In most of these paradigms the mutants show some memory immediately after training, but the memory decays more quickly than in control flies (Duerr and Quinn, 1982; Dudai, 1983; Tempel et al., 1983; Tully and Quinn, 1985), indicating defects in memory processes for different types of conditioned behavior.

Molecular information on the genes disrupted by these mutations is available only for three mutants, dunce, rutabaga, and $D C O$. It is striking that they code for molecules that are involved in the cAMP signaling pathway-dunce codes for cAMP phosphodiesterase (PDE) (Byers et al., 1981; Davis and Kiger, 1981; Chen et al., 1986; Qiu et al., 1991), rutabaga codes for a $\mathrm{Ca}^{2+} /$ calmodulin-responsive adenylyl cyclase (Livingstone et al., 1984; Levin et al., 1992) and DCO codes for cAMP dependent protein kinase (Skoulakis et al., 1993). Molecules of general signaling pathways are likely to be involved in various cellular

\footnotetext{
Received June 10, 1994; revised Nov. 11, 1994; accepted Nov. 16, 1994.

We thank E. M. C. Skoulakis, A. J. Nighorn, M. Capovilla, and R. W. West for reading the manuscript. This work was supported by fellowships from the Swiss National Science Foundation and the Janggen-Pöhn Stiftung to B.D. and an NIH grant to R.L.D.

Correspondence should be addressed to Ronald L. Davis, Baylor College of Medicine, Department of Cell Biology, One Baylor Plaza, Houston, TX 77030.

"Present address: UT MD Anderson Cancer Center, Department of Molecular Genetics, Box 45, 1515 Holcombe Boulevard, Houston TX 77030.

Copyright (C) 1995 Society for Neuroscience $0270-6474 / 95 / 153490-10 \$ 05.00 / 0$
}

processes in many cell types. Mutations in their genes can therefore lead to pleiotropic effects that make the separation of specific physiological roles difficult. For example, $d n c$ has been shown to affect female fertility (Byers et al., 1981; Salz et al., 1982; Bellen et al., 1987) as well as the morphology and plastic electrophysiological properties of the larval neuromuscular junction (Corfas and Dudai, 1991; Delgado et al., 1991; Zhong and Wu, 1991; Zhong et al., 1992).

The diversity of $d n c$ transcripts may account partly for the observed pleiotropy. The gene encodes at least 10 different RNA transcripts from at least five different transcription start sites (Qiu et al., 1991). Using chromosomal deletions and inversions, Qiu and Davis (1993) found separate functions for the various transcriptional units. For example, one transcriptional unit appears primarily responsible for elevated PDE expression in mushroom bodies (see below) and memory. Others are required for immediate learning and female fertility.

The dunce protein is expressed preferentially in the neuropil of the mushroom bodies and at low levels in the neuropil of the remainder of the nervous system. This observation is consistent with a role for dunce in olfactory learning and memory processes since the mushroom bodies have long been implicated as principle centers mediating olfactory learning in insects (Erber et al., 1980; Heisenberg et al., 1985; Schildberger, 1984; de Belle and Heisenberg, 1994). However, a firm physiological role for dunce has not been demonstrated. Rather, the mutants may learn poorly due to structural defects in the nervous system arising from a developmental requirement for the gene. Indirect evidence for a physiological involvement of PDE in learning has been obtained previously. Feeding PDE inhibitors to adult flies resulted in a reduction of learning (Folkers and Spatz, 1984; Dudai et al., 1986).

We addressed the possible physiological role of the dunce PDE in learning/memory and female sterility directly by introducing sequences coding for a heat inducible dunce minigene into mutant $d n c$ flies. In addition, we were interested in determining whether a CDNA for a rat dunce homologue (Davis et al., 1989) could substitute functionally for the Drosophila gene. We report here that expression of the Drosophila gene shortly after eclosion confers partial rescue of the $d n c$ female sterility. Moreover, induction of Drosophila as well as rat dunce minigenes shortly before training significantly improves the learning/ memory of $d n c$ mutants. The results show functional conservation between the Drosophila dunce gene and its rat counterpart and demonstrate the participation of PDE products in the physiology of learning/memory.

\section{Materials and Methods}

$P$-Factor constructs

A $1.75 \mathrm{~kb}$ dunce cDNA clone spanning the carboxy terminal part of the open reading frame [containing the last 19 nucleotides of exon 5 
exons 6-12 and exon 13 up to the stop codon (Qiu et al., 1991)] was cloned into the vector p70ATG (Petersen and Lindquist, 1989). This vector contains 1140 bp of $5^{\prime}$ flanking and 250 bp of leader sequences of the Drosophila hsp 70 gene. The dunce cDNA clone was inserted between a BamH 1 site created by mutation of the hsp70 ATG start codon (Petersen and Lindquist, 1989) and a Sall site 6 bp $5^{\prime}$ to the hsp70 stop codon. The Sall site is followed by 155 bp of trailer and 75 bp 3' flanking sequences from the hsp 70 gene. The hspdnc cassette was subsequently cloned into the Drosophila transformation vector, CaSpeR4, which contains a white ${ }^{+}$minigene as a selectable marker (Pirrotta, 1988), to yield a vector denoted as hspdnc in the text. A transformation vector (hspRD1) carrying a rat cDNA homolog of dunce, named RDI (Henkel-Tigges and Davis, 1990), was constructed in an identical fashion. Transformants were generated by P-element mediated transformation (Spradling and Rubin, 1982) of $D f(l) y w^{67023}$ (w) embryos using the "turbo" helper plasmid pUChsp $\Delta 2-3$ wc (Tomlinson et al., 1988); a gift from E. Hafen).

For rescue of the embryonic lethality caused by microinjection of the transformation constructs, the PDE inhibitors rolipram (Henkel-Tigges and Davis, 1990) and SQ20009 were used at a concentration of 100 $\mathrm{mM}$ in the injection buffer. Rolipram was a gift from Schering $\mathrm{AG}$, SQ20009 a gift from Squibb, Inc.

Transformed flies were identified using the white ${ }^{+}$marker and the insertions mapped to chromosomes with the use of a $w ; C y O ; T M 3$ balancer stock. The transposons hspdnc26, hspdnc54, and hspRDl-12 were mapped to the $2 \mathrm{nd}$, $3 \mathrm{rd}$, and 2 nd chromosomes, respectively. Flies heterozygous for the transposon and the appropriate balancer were crossed to obtain flies homozygous for the transposons.

\section{Genetics}

Flies were kept on standard cornmeal/molasses based food or later (experiment using flies with normalized background) on standard cornmeal/sugar based food.

Transposons of independent transforned lines were introduced into dnc mutants as follows. The parental $d n c$ chromosome was kept balanced with $F M 7 a$ throughout the crosses and in all stocks. Parental $d n c /$ $F M 7 a$ females were crossed to $w$; CyO; TM3 males. The resulting $w /$ $F M 7 a ; C y O$ or $w / F M 7 a ; T M 3$ females were backcrossed to $d n c$ males to obtain $d n c / F M 7 a$ females heterozygous for a second or third chromosome balancer. They were crossed to $w$-hspdnc or $w$-hspRDI males and $w / F M 7 a$ progeny females heterozygous for transposon and balancer were collected and backcrossed to their $d n c$ brothers heternzygous for the transposon and the balancer. Lines homozygous for the transposons were established from progeny in the subsequent generation.

The hspdnc and hspRD1 12 transformants in the dnc mutant background generated from the above scheme contain a nonuniform genetic background, with chromosomes from both the dnc stock and the $D f(I) y$ $w^{677_{23} 3}$ stock. We nornalized the genetic background for some experiments by keeping the dnc chromosome balanced at all times and sequentially replacing the autosomes with chromosomes originating from the $D f(I) y w^{6 / c 2,3}$ stock using $C y O$ and $T M 3$ balancer chromosomes. For second chromosome transposons, the $d n c$ stock was made homozygous for the $3^{w}$ chromosome (3rd chromosomes from the $D f(1) y w^{67 \mathrm{c} 23}$ stock) using the procedure described above. This resulted in a $d n c / F M 7 a ; 2$; $3^{\mathrm{w}} / 3^{\mathrm{w}}$ stock. The $d n c / F M 7 a$ females from this stock were crossed to $w ; 2^{w} / \mathrm{CyO} ; 3^{w} / \mathrm{TM} 3$ balancer males and $d n c ; C y O / 2 ; 3^{w /} / 3^{w}$ males of the next generation mated to their $w / F M 7 a ; C y O / 2 ; 3^{w} / 3^{w}$ female siblings. The resulting dnc/FM7a;CyO/2; $3^{\mathrm{w}} / 3^{\mathrm{w}}$ females were then mated with $w$-hspdnc males homozygous for the insert on the second chro mosome. The $w / F M 7 a ; C y O / h s p d n c ; 3^{w} / 3^{w}$ progeny females were crossed to their $d n c ; C y O /$ hspdnc; $3^{*} / 3^{*}$ brothers and a $d n c / F M 7 a$; hspdnc/hspdnc; $3^{w / 3} 3^{w}$ stock established. The original dnc mutants and their parental chromosomes $\left(y w f^{36 \mathrm{a}}\right.$ and $C S$ for $d n c^{\mathrm{ML}}$ and $d n c^{1}$, respectively) were normalized in a similar fashion, keeping the X-chromosomes intact over $F M / a$ throughout the scheme. An analogous procedure was used for 3 rd chromosome transposons.

\section{Phosphodiesterase assay}

Ten to 20 flies were heat shocked in prewarmed food vials in a $37^{\circ} \mathrm{C}$ water bath for $15 \mathrm{~min}$, weighed and homogenized at different time points after heat shock. Homogenates were frozen in a dry ice-ethanol bath, stored at $-70^{\circ} \mathrm{C}$ and assayed as described by Davis (1988). The assay mixture contains an excess of unlabeled cGMP which competitively inhibits the calcium-calmodulin activated cyclic nucleotide phos-

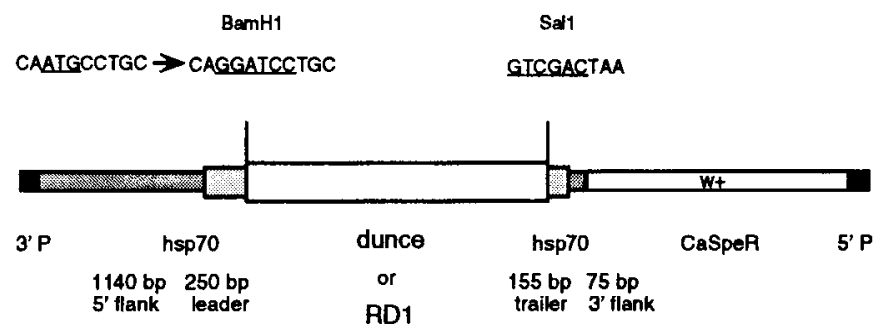

Figure 1. Structure of the hspdnc and hspRD1 transformation vectors The cDNAs encoding Drosophila dunce ${ }^{+}$or rat dunce-like $(R D I)$ cAMP PDE activity were placed between Drosophila hsp70 $5^{\prime}$ and $3^{\prime}$ controlling elements using the BamHI and Sall sites. Each cassette was then inserted into the transformation vector, CaSpeR. The BamHl site was created by mutagenesis of the hsp 70 ATG translation start codon (Petersen and Lindquist, 1989). The Sall site within the hsp70 gene is located 6 bp $5^{\prime}$ of the hsp 70 stop codon, but the Drosophila and rat cDNAs both contain their own stop codon. The transformation vector, CaSpeR, carries the white minigene $\left(\mathrm{w}^{+}\right)$as a selectable marker and the inverted repeats required for integration $\left(5^{\prime} P\right.$ and $3^{\prime} P$, respectively).

phodiesterase (Davis, 1988). The dunce PDE is specific for cAMP as substrate and is unaffected by cGMP in the assay cocktail. Rolipram or SQ20009 was added in some assays to $100 \mathrm{~mm}$ or $140 \mathrm{~mm}$ final concentration, respectively. Assays were performed in duplicate with the two measurements averaged to produce one data point (see figure captions).

\section{Learning/memory tests}

Since the $d n c$ chromosome was balanced over $F M 7 a$, $d n c$ males were individually selected for training. Two to $5 \mathrm{~d}$ old flies were collected under $\mathrm{CO}_{2}$ anesthesia and kept in food vials for 12-14 hr before the training. For most experiments, vials were coded by personnel not involved in the experiment so that heat shock and training were performed blind.

Heat shock. About 150 flies were heat shocked in each prewarmed food vial in a $37^{\circ} \mathrm{C}$ water bath, left to recover for $2 \mathrm{hr}$ at room temperature, and heat shocked again for $15 \mathrm{~min}$. After each heat shock, the vials were allowed to cool to room temperature and the flies were transferred to a fresh food vial. Ninety minutes after the second heat shock the vials were transferred to the darkened training room for adaptation. Training began $30 \mathrm{~min}$ later.

Training and testing. A modified version of the classical conditioning apparatus (Tully and Quinn, 1985) was used. Training was performed in a Plexiglas shock tube $(7.6 \mathrm{~cm}$ long with a $1.2 \mathrm{~cm}$ diameter, i.d.) lined with a $4.2 \times 7.6 \mathrm{~cm}$ flexible copper grid, etched on either Teflon or Kapton. Silicon rubber tubing secured nylon netting at both ends and attached the Plexiglas training tube to plastic tubing connectors. One end was connected to the house vacuum and the other to silicon rubber tubing attached to a bubbling flask (Fisherbrand 11-184). Odors were generated by sucking room air over the surface of $1 \mathrm{ml}$ of 3-octanol (OCT) or $1 \mathrm{ml}$ of 4-methylcyclohexanol (MCH) [ICN K\&K Laboratory] contained in the bubbling flasks, through the silicon rubber tubing to the training tube and out through the vacuum source.

A group of $100-150$ flies were sequestered in the training tube. After 1 min. presentation of room air, $\mathrm{MCH}$ odor was presented to the flies for $1 \mathrm{~min}$ simultaneous with $121.25 \mathrm{sec}, 90 \mathrm{~V}$ square-wave pulses at $5 \mathrm{sec}$ intervals. After washing the tube with room air for $1 \mathrm{~min}$, OCT odor was presented to the flics for $1 \mathrm{~min}$ without shock. Air flow through the training tube was $550 \mathrm{ml}$ per minute. For testing, the flies were transferred immediately into the sliding central compartment of a choice chamber (the design of the apparatus is available from the authors) and rested for $1 \mathrm{~min}$. The choice chamber has a circular central compartment of $2.1 \mathrm{~cm}$ diameter and $0.5 \mathrm{~cm}$ width which houses the flies and contains a port for applying vacuum. The sliding compartment is pushed to a point between two arms, each $2.1 \mathrm{~cm}$ in diameter and $10.5 \mathrm{~cm}$ in length, with each arm connected via tubing to a bubbling flask containing either OCT or MCH. The air flow was adjusted and monitored at a rate of $1750 \mathrm{ml}$ per min per arm. Flies were given 2 min to disperse from the central compartment and into the two collection arms. The training and testing procedure was repeated with a new group of flies, with OCT being paired with electric shock and $\mathrm{MCH}$ 
A

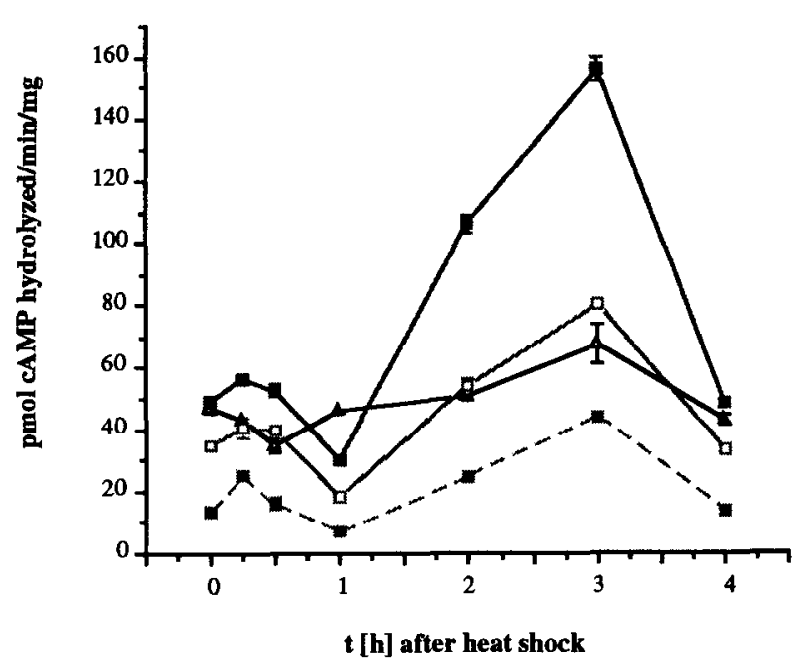

B

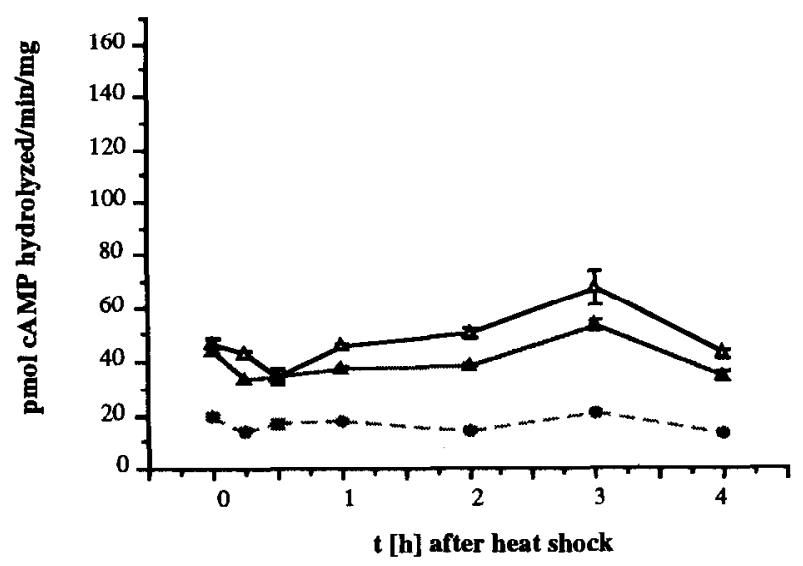

C

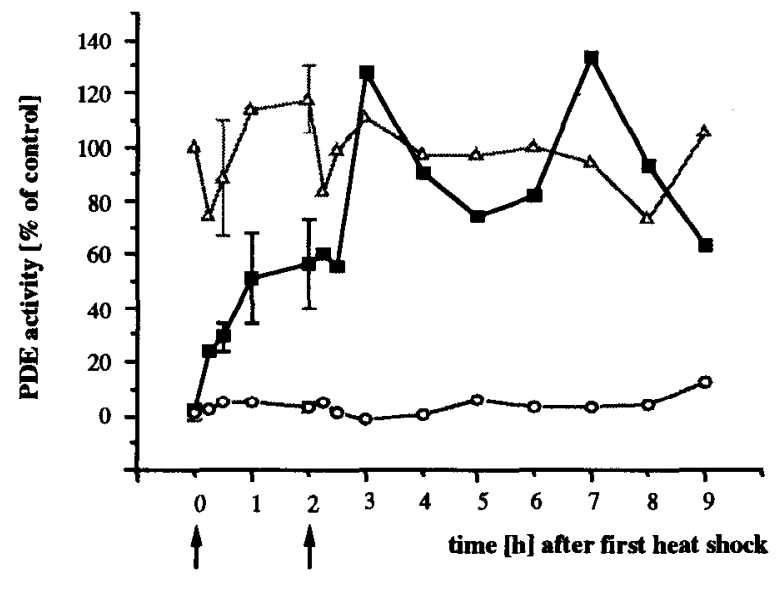

D

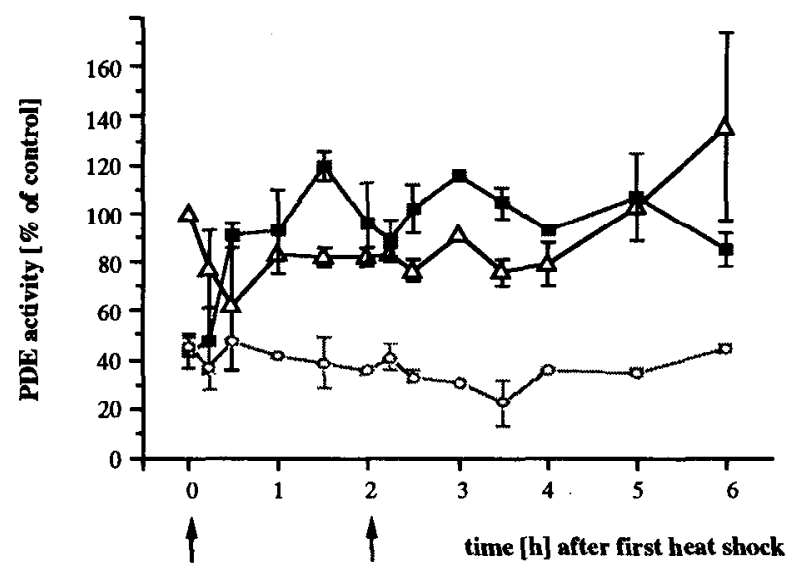

E

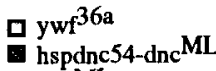

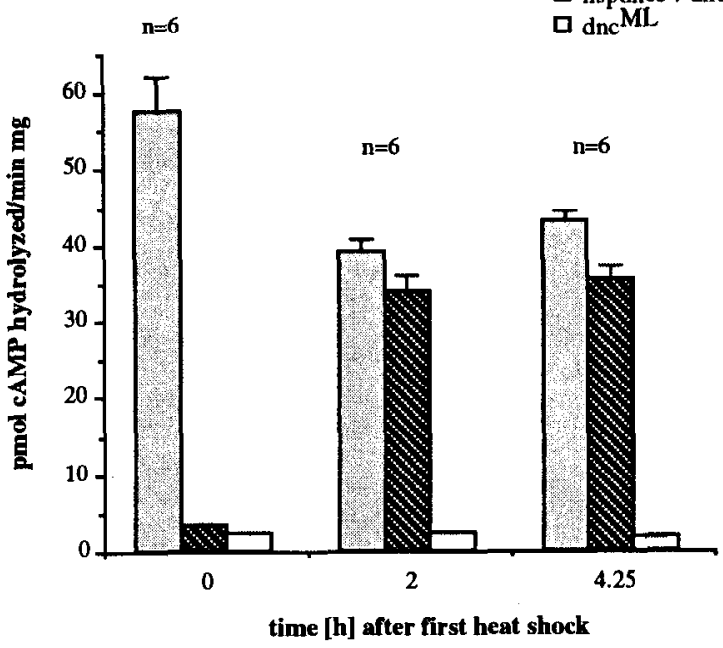

Figure 2. Time course of heat-induced cAMP PDE activity encoded by the Drosophila or the rat transposons. Flies were heated in a $37^{\circ} \mathrm{C}$ water bath in prewarmed vials for 15 min once or twice and homogenized at various times afterwards. cAMP PDE activity was measured in whole fly 
serving as control. These procedures were carried out at $23^{\circ} \mathrm{C}$ in $\operatorname{dim}$ red light.

The learning index of each group of flies was calculated as the fraction of flies avoiding the shock-associated odor minus the fraction of flies avoiding the control odor (Quinn et al., 1974). One index is the average of the scores of the two groups of flies, one shocked in the presence of $\mathrm{MCH}$, the other shocked in the presence of OCT.

For later experiments (testing flies with a normalized background) a semiautomated training apparatus developed by C. Chromey and R.D. was used. The procedures were performed in an environmental room at $25^{\circ} \mathrm{C}$ and $70 \%$ relative humidity in dim red light. The training was automated using a three-way valve (Series 18 Valves, General Valve. Fairfield, NJ) through which air and the two odorants were dispensed, the valve ports being connected to bubbling flasks containing odorants as described above. A programmable controller (Sysmac C20, Omron Corp., Schaumburg, IL) regulated the opening and closing of the valves as well as the delivery of the shock pulses concurrent with one of the odorants. Two groups of flies were trained simultaneously using two training tubes set in parallel, one for training with $\mathrm{MCH}$, the other for training with OCT. The training protocol consisted of the delivery of air for $30 \mathrm{sec}, 1 \mathrm{~min}$ of the first odor concurrent with $11,1.25 \mathrm{sec}, 90$ $\mathrm{V}$ square-wave pulses (the first shock beginning exactly $5 \mathrm{sec}$ after odor presentation), air for $30 \mathrm{sec}$, the control odor for $1 \mathrm{~min}$ (no shock), and another air purge for $30 \mathrm{sec}$. Air flow through the training apparatus was monitored with a flow meter and set to $550 \mathrm{ml} / \mathrm{min}$. Testing remained as described above.

\section{Female fertility tests}

The flies were kept in food vials in an incubator (Hotpack programmed refrigerated incubator) programmed to cycle continuously between $25^{\circ} \mathrm{C}$ $(2 \mathrm{hr})$ and $37^{\circ} \mathrm{C}(1 \mathrm{hr})$. The vials were kept in a small amount of water to avoid desiccation. Virgin $d n c$ or control $y w f^{36 a}$ females, less than 2 hr old, were subjected to cycling conditions for $4 \mathrm{~d}$ in the absence of males. A second set of females were kept at $23^{\circ} \mathrm{C}$ for the same period. The females were then individually placed with three Canton-S males for $1.5 \mathrm{~d}$ under cycling conditions, or at $23^{\circ} \mathrm{C}$. After removing the parents, the vials were kept at $23^{\circ} \mathrm{C}$ and the cmerging progeny scored 20 $\mathrm{d}$ after mating. The $1.5 \mathrm{~d}$ mating/egg laying period was chosen from pilot experiments which indicated that heat shock of transformed larvae is lethal within a time window during late first instar.

\section{Statistical analysis}

Analyses were performed on a MacIntosh IIci with JMP 2.0 statistical software (SAS Institute, Inc.). The significance of the transposon-heat shock interaction was determined by two-way analysis of variance. Comparisons between groups were by unplanned multiple comparisons using Tukey-Kramer analysis at $\alpha=0.05$. Data from each pair of nonheat-shocked versus heat shocked flies of the same genotype were analyzed by planned pairwise comparison.

\section{Results}

Injection of Drosophila dunce or rat dunce-like minigenes under the control of a heat shock promoter is lethal to embryos

The control sequences of the Drosophila hsp 70 gene responsible for fast heat inducibility were used to prepare constructs that express Drosophila dunce or rat dunce-like cAMP PDE conditionally (hspdnc and hspRD1, respectively; see Fig. 1). The cDNA used for the Drosophila hspdnc construct encodes a portion of the dunce protein which is common to all predicted isoforms (Qiu et al., 1991) and contains the conserved domain shared with many other types of PDEs (Chen et al., 1986; Beavo and Reifsnyder, 1990). Similar cDNAs when expressed in yeast produce cAMP PDE activity indistinguishable in kinetics from the dunce-encoded activity in whole fly homogenates (Qiu et al., 1991). The rat cDNA RD1 was previously isolated by crosshybridization to a Drosophila dunce cDNA (Davis et al., 1989). When expressed in yeast RDl produced cAMP PDE activity with kinetic properties very similar to the Drosophila enzyme (Henkel-Tigges and Davis, 1990). The hspdnc and hspRDl constructs were cloned into the CaSpeR P-element based transformation vector for the generation of transgenic flies by germline transformation of $D f(I) y w^{67 \mathrm{c} 2.3}$ flies (abbreviated "w" throughout the text).

Surprisingly, all of the embryos injected with these transformation constructs died. Close examination revealed that development in the injected embryos was arrested before or at the onset of cellular blastoderm with a characteristic phenotype affecting primarily the posterior of the embryo (not shown). We did not characterize the lethality of these embryos further, but tentatively concluded that transient PDE expression from the injected constructs interfered with cAMP levels and/or distribution and that this resulted in lethality. Others have shown that P-element constructs show transient expression upon injection (Steller and Pirrotta, 1984). Moreover, the observed phenotype is consistent with the previous observation that embryonic development is disrupted from defects primarily at the posterior end of embryos upon manipulation of the maternal genotype to alter embryonic cAMP content (Whitehouse-Hills et al., 1992). We therefore attempted to rescue the lethality by coinjecting a PDE inhibitor with each transformation construct. Rolipram is a potent PDE inhibitor that has been shown to inhibit the rat RD1 PDE but not the Drosophila enzyme (Henkel-Tigges and Davis, 1990). Coinjection of rolipram with the RD1 transformation construct rescued the embryonic lethality and allowed for the recovery of transformed w-hspRD1 flies. The PDE inhibitor SQ20009 was used for coinjection with the Drosophila construct. This allowed for the recovery of w-hspdnc transformed flies. More than 10 independent transformed lines were recovered for each construct.

\section{The Drosophila and the rat transposons are conditionally expressed in transformed flies after heat shock}

Transformants containing two copies of the transposon (whspdnc or w-hspRD1) were subjected to heat treatment and as-

\section{$\leftarrow$}

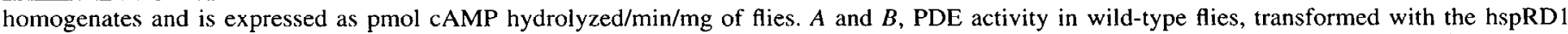

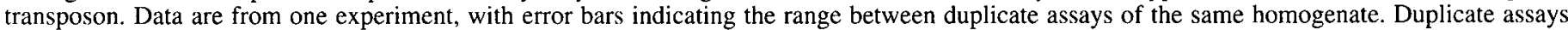

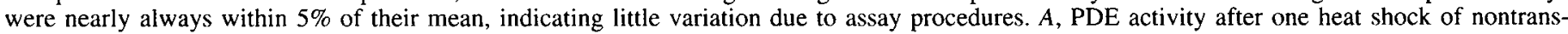

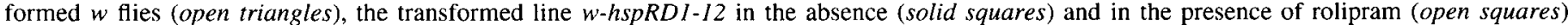

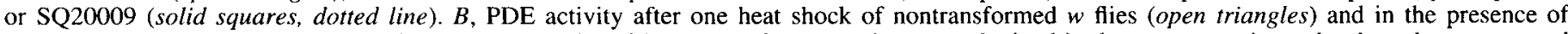

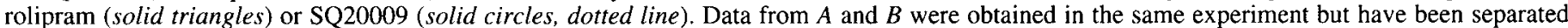

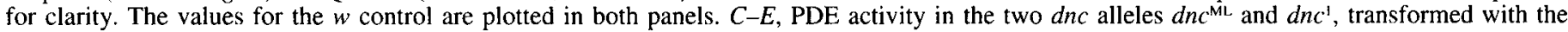

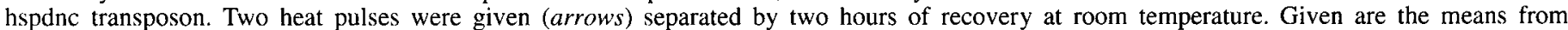

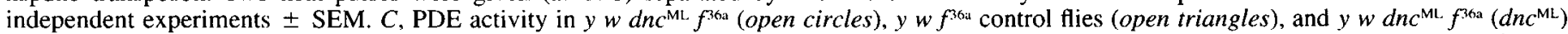

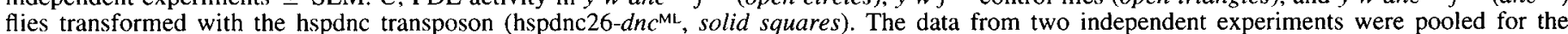

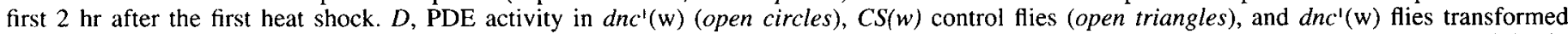

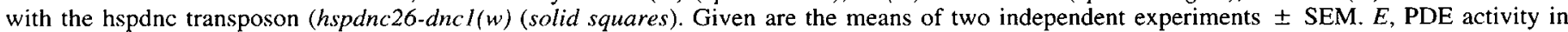

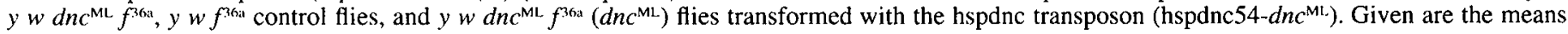
\pm SEM. 
sayed for induced PDE activity. Pilot expcriments indicated that a 15 min heat shock was sufficient to induce the transposonencoded enzyme. This mild heat shock condition does not interfere with the behavior of control flies in learning assays (see below) and was therefore used throughout these experiments. Figure $2 A$ shows the time course of PDE induction of hspRD1 after one $15 \mathrm{~min}$ heat shock in a transformed line containing two copies of the RD1 transposon in addition to the endogenous dunce gene (w-hspRD1-12). PDE activity fluctuates in control (w) and transformed flies after heat shock (Fig. $2 A, B$ ). It drops to a low point at $1 \mathrm{hr}$ after heat shock in the transformants and then peaks at twice the control level at $3 \mathrm{hr}$. To determine whether the induced activity was due to the expression of the rat PDE, we assayed the homogenates in the presence of rolipram. Rolipram had little effect on the endogenous activity from $w$ flies (Fig. $2 B$ ), but it reduced the activity of the w-hspRD1-12 flies to that in control flies. The induced activity observed in the transformants is therefore due to the expression of the transposon-encoded rat enzyme. Addition of the less-selective PDE inhibitor SQ20009, reduced the activity of the endogenous as well as the transgenically supplied enzyme (Fig. $2 A, B$ ).

Chromosomes carrying the hspRDl or hspdnc transposons were introduced into two different $d n c$ mutants, $d n c^{1}$ and $d n c^{\mathrm{MI}}$, and PDE activity was measured in the mutant backgrounds after heat shock. The mutant $d n c^{1}$ was isolated in a screen for strains deficient in learning (Dudai et al., 1976). The mutant $d n c^{\mathrm{ML}}$ was isolated in a screen for mutants with low cAMP PDE activity (Davis and Kiger, 1981). Both mutants show the learning/memory deficits that are characteristic for $d n c$ mutants (see below).

Figure $2 C$ compares the time course of PDE induction in flies carrying an hspdnc transgene (hspdnc26-dnc $c^{\mathrm{ML}}$ ). Two heat shocks separated by a two hour recovery period were used to determine whether higher levels of PDE activity could be achieved by repetition. The $d n c^{\mathrm{MI}}$. mutants had a complete absence of activity at all time points, but hspdnc $26-d n c^{\mathrm{Ml}}$ - flies showed rapidly induced activity after the first heat shock. The second heat shock led to a further increase in transgene encoded enzyme activity, reaching and maintaining the levels found in the parental strain for at least $6 \mathrm{hr}$. PDE induction in $d n c^{1}$ mutants carrying two copies of the hspdnc or hspRD1-12 transposons showed the same charactcristics of expression described for the $d n c^{\mathrm{ML}}$ mutants [shown in Figure $2 D$ for hspdnc26-dncl(w)]. The $d n c^{\prime}$ mutant retains about $46 \%$ of the wild-type PDE levels, which increase about 2.5 times in heat shocked transgenic flies to reach and at some time points exceed, wild-type levels. Figure $2 E$ compares the induction of PDE activity at two hours after the first heat shock and two hours after the second heat shock in $d n c^{\mathrm{ML}}$ mutants canying another hspdnc transgene (hspdnc54-dnc $c^{\mathrm{ML}}$ ). Higher levels of activity in parental $y w f^{36 a}$ flies in the absence of heat shock over these found at 2 or $4.25 \mathrm{hr}$ likely reflect the mild fluctuations in activity that we have observed in control and transformed lines. Activity in transformed $h s p d n c 54-d n c^{\mathrm{ML}}$ flies is indistinguishable from the mutant in the absence of heat shock. In contrast to transformant line 26, wild-type levels are reached $2 \mathrm{hr}$ after the first heat shock and are maintained at this level $2 \mathrm{hr}$ after the second heat shock (Tukey-Kramer, $\alpha=0.05$ ).

In summary, the transposon encoded rat and Drosophila PDE activity, introduced into either a wild-type background or two different $d n c$ mutants, is consistently induced after heat shock. The induced activity in some transformants reaches wild-type levels after one heat shock and persists for several hours after heat treatment. This induction is superimposed upon minor fluc- tuations in endogenous activity due to heat shock. However, since PDE activity was measured in whole fly extracts, the levels of activity in those cells important for learning/memory are likely to be different in wild-type and heat-treated transgenic flies. For example, since the hsp70 promoter confers expression in nearly all cells (Bonner and Pardue, 1976; Bonner et al., 1984; Lis et al., 1983), the concentration of the induced PDE in mushroon body cells, the preferred site of dunce expression, is likely to be considerably lower than in wild type.

Expression of the Drosophila or the rat enzyme shortly before training partly rescues the dnc learning deficit

Mutant flies carrying a transposon were tested in an olfactory classical conditioning paradigm with and without heat shock (see Materials and Methods). This learning situation pairs the presentation of a specific odor with electrical shock for training, while a second odor not coupled to shock serves as the control. The subsequent avoidance behavior of the flies to the shockassociated odor is used as a measure of learning. Most wildtype flies avoid the shock-associated odor after training, whereas dnc flies show much less avoidance of the shock-associated odor (see below). We define the degree of avoidance obtained immediately after training as learning, although 3 min elapse between the training and the actual testing (see Materials and Methods). Therefore, the learning index reflects acquisition, retrieval, and immediate memory. The hehavioral experiments described generally followed a balanced design, in which all flies were reared, selected, handled and trained in parallel in each training session. Therefore, the relevant controls for each set of data are contained within each figure.

The $y w f^{36 a}$ flies, the parental genotype for the $d n c^{\mathrm{Ml}}$ mutant, exhibited learning scores of about 0.4 immediately after training either with or without prior heat shock (Fig. $3 A$ ). Heat shock also had no significant effect on the low scores $(0.15)$ of the mutant, $d n c^{\mathrm{ML}}$. In contrast, the scores obtained after heat shock of $d n c^{\mathrm{MI}}$ transgenic lines carrying the Drosophila transposon, hspdnc26 and hspdnc54, as well as the rat transgene hspRD112 , were significantly higher than those of the $d n c^{\mathrm{MI}}$ mutants or non-heat-shocked, but transgenic siblings. The hspdnc26 and hspdnc54 transposons did not provide complete rescue, since the scores of heat-shocked flies carrying these transposons were lower than the score of the parental $y w f^{36 a}$ flies. However, the learning score of hspRD1-12 carrying $d n c^{\mathrm{ML}}$ flies was indistinguishable from the $y w f^{36 a}$ control. The slightly elevated scores of hspdnc54- $d n c^{\mathrm{ML}}$ flies in the absence of heat shock over the $d n c^{\mathrm{ML}}$ control are only marginally significant. It is possible that, if real, this represents a leakiness of the hsp 70 promoter, which has a low level of activity in the absence of heat shock that has been shown to be capable of providing rescue when driving other genes (Steller and Pirrotta, 1985; Boggs et al., 1987; Bishop and Corces, 1988). This could suggest that learning ability is very sensitive even to low levels of functional dunce protein expected from such leakiness of the hs promoter. However, the failure to detect this leakiness in biochemical assays (Fig. 2) may suggest that the behavioral assay is more sensitive than the biochemical one. Similar observations of rescue without heat shock have been reported for hsp-per transformants in rescuing circadian rhythms (Ewer et el., 1990).

To eliminate the possibility that behavioral rescue was specific to the $d n c^{\mathrm{ML}}$ allele, we crossed some of the transposons into $d n c^{\mathrm{l}}$ mutants. The $d n c^{1}$ flies carrying either the hspdnc54 or the hspRD1-12 transposon performed significantly better after heat 
$\mathbf{A}$

\section{Learning Index}

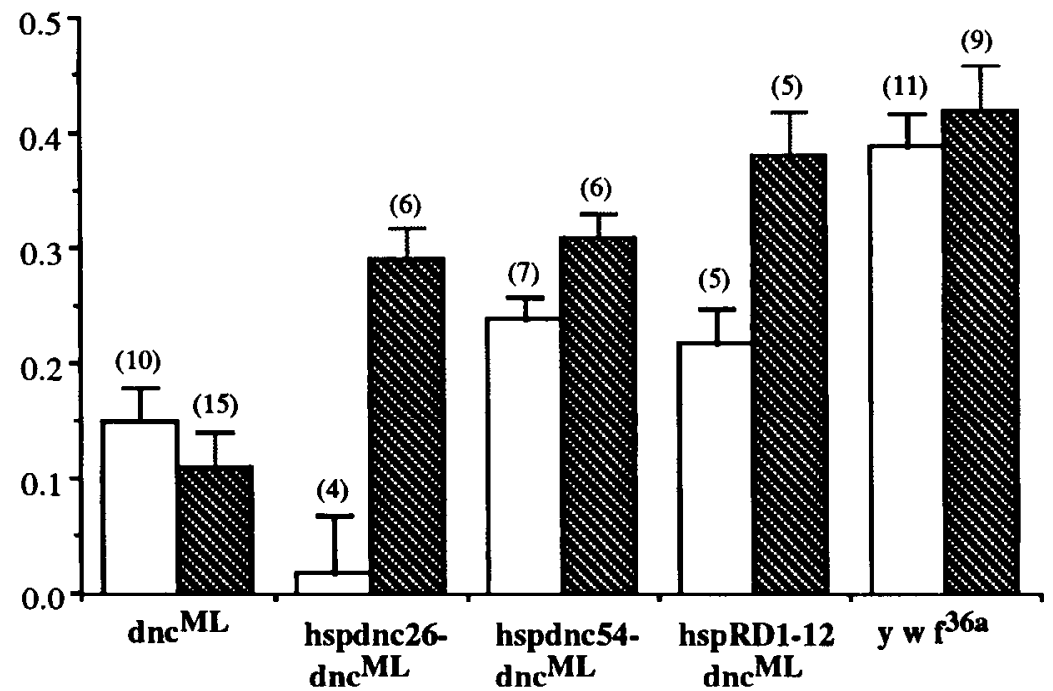

B

\section{Learning Index}

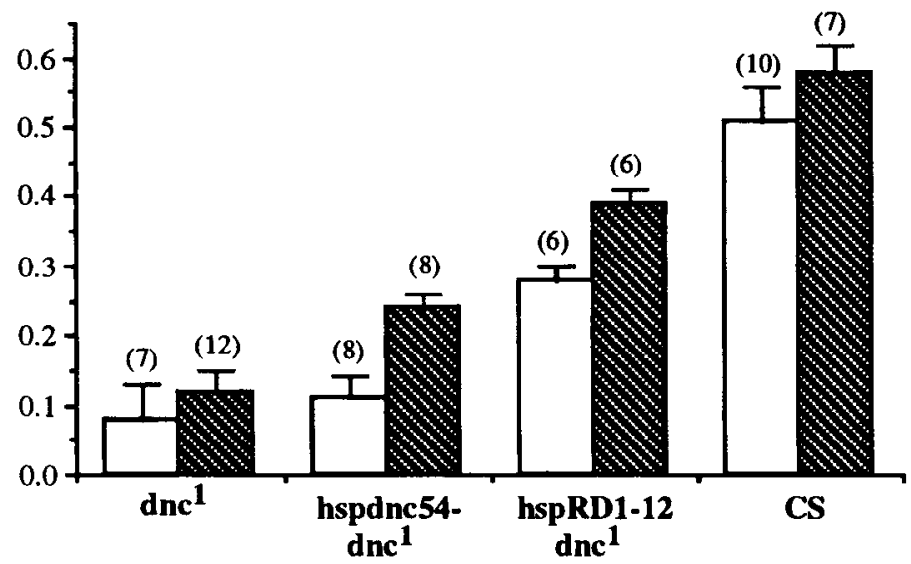

Figure 3. Rescue of the learning deficit of $d n c$ flies with the Drosophila hspdnc and the rat hspRD1-12 transposons. $A$, The learning indices ( \pm SEM) of $y w f^{\text {36a }}$ control flies and $y w d n c^{\mathrm{ML}}$ $f^{36 a}\left(d n c^{\mathrm{ML}}\right)$ flies with and without the transposons are graphed. Open bars indicate scores obtained without heat shock, solid bars indicate those obtained after heat-shocking the flies 2 and $4 \mathrm{hr}$ prior to training. A learning index of 1.0 represents perfect learning; 0 represents no learning. The learning index of non-heat-shocked $h s p d n c 26-d n c^{\mathrm{ML}}$ is not significantly different from non-heat-shock $d n c^{\mathrm{ML}}$ flies, but appears artificially low due to the sample size. $B$, The learning indices $( \pm$ SEM) of Canton-S (CS) control flies and $d n c^{\prime}$ flies with and without the transposons. Numbers in parentheses indicate the number of repetitions. shock than $d n c^{\prime}$ flies or $d n c^{\prime}$ flies with the transposons but without heat shock (Fig. 3B). The increase in scores after heat shock, however, was less impressive with this mutant than with $d n c^{\mathrm{ML}}$. This may indicate that the level of rescue is dependent upon the nature of the endogenous allele (see Discussion). The hspRD112 flies showed partial rescue in the absence of heat shock which increased significantly further after heat shock.

It has been reported that genetic background may influence learning performance (Dudai, 1977; Tully and Quinn, 1985; Gailey et al., 1991, Dura et al., 1993). In the course of combining chromosomes containing the transposons with those carrying $d n c$ alleles, the genetic background was changed compared to the parental line. To determine whether the genetic background differences may have affected our results, we repeated some of the experiments after normalizing the genetic background of the transformed and control flies (see Materials and Methods). Consistent with our previous results, we observed partial rescue of the $d n c^{\mathrm{ML}}$ and the $d n c^{\prime}$ alleles (Fig. 4) with the characteristics observed in the non-out-crossed lines. As before, $d n c^{\mathrm{Ml}}$. trans- genic flies showed a more pronounced increase in learning after heat shock than $d n c^{\prime}$ transgenic flies. Some quantitative differences in rescue were evident after background normalization with the ovcrall learning indices being higher than in previous experiments. This could be due to altered genetic background or to a switch to an automated version of the training apparatus (see Materials and Methods) during the course of these experiments. The hspdnc26 transposon conferred appreciable rescue to the $d n c^{\mathrm{ML}}$ allele, although it was incomplete as before. Heatshock treatment of $d n c^{1}$ flies containing the hspdnc 26 transposon produced marginal rescue (not shown). With both the hspdnc26 and hspdnc54 transposons, significant rescue was obtained.

In summary, both sets of results show that the learning deficit of $d n c$ mutant flies can be ameliorated by the conditional expression of transposon borne Drosophila or rat dunce minigenes.

The female sterility of $\mathrm{dnc}$ females can be partially rescued by conditional expression of the hspdnc transposon

We next addressed the question of whether the female sterility of $d n c$ flies can be rescued by heat-induced expression of the 
A

\section{Learning Index}

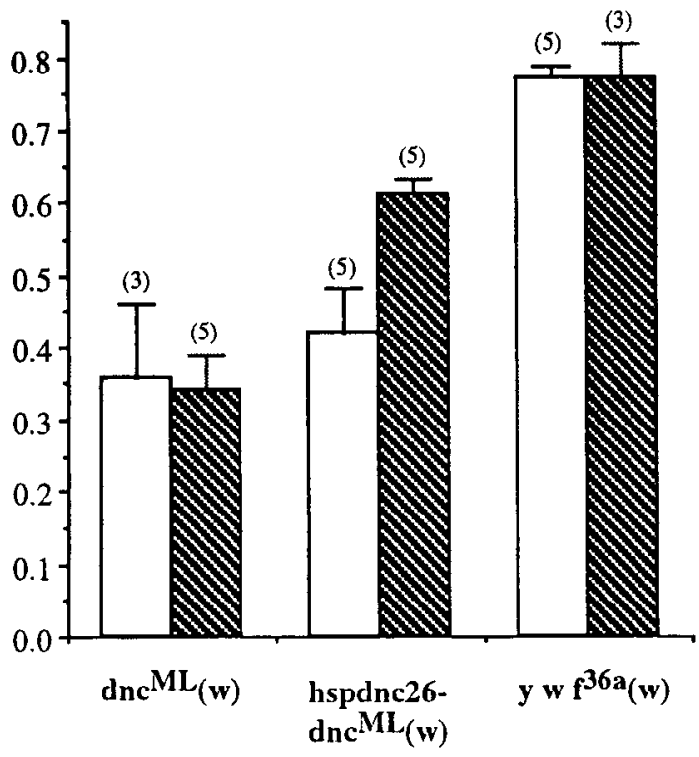

B

\section{Learning Index}

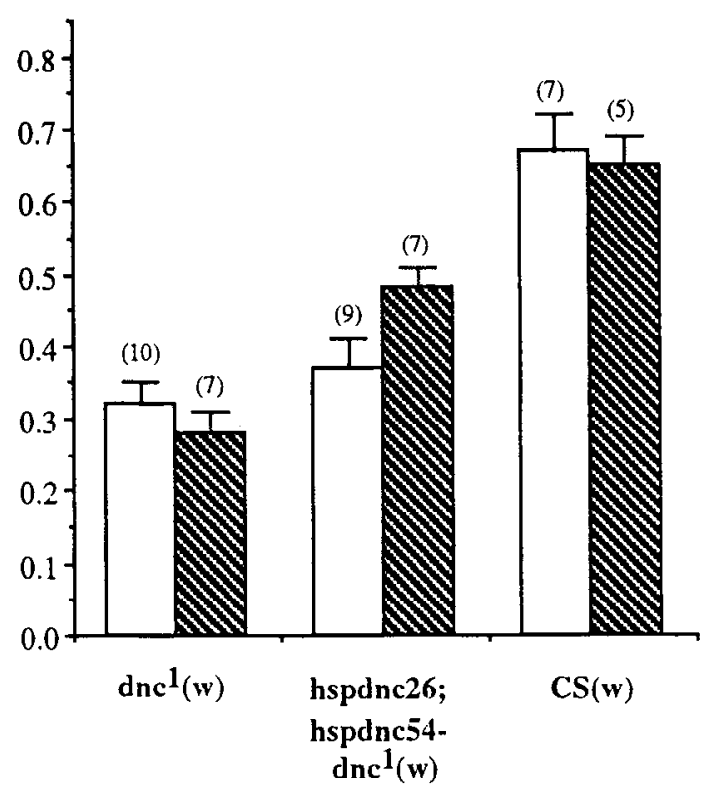

Figure 4. Rescue of the learning deficit of $d n c$ flies with normalized genetic background. All strains were normalized to the $D f(1) y w^{67.23}$ genetic background which is indicated by the designation $(w)$. Open bars indicate scores obtained without heat shock; solid bars indicate those obtained after heat shocking the flies 2 and $4 \mathrm{hr}$ prior to training. $A$, Learning indices of heat shocked and non-heat shocked $y w d n c^{\mathrm{ML}} f^{36 \mathrm{a}}, d n c^{\mathrm{ML}} \mathrm{flies}$, the $y w f^{36 \mathrm{a}}$ control, and $y w d n c^{\mathrm{ML}} f^{36 \mathrm{a}}$ flies carrying one copy of the Drosophila transposon hspdnc (hspdnc26-dnc $\mathrm{c}^{\mathrm{ML}}$ ). B, Learning indices of heat shocked and non-heat-shocked $d n c^{\prime}$ flies carrying two copies of the transposon hspdnc26 and two of hspdnc54 (hspdnc26;hspdnc54-dnc') along with the control strains $d n c^{1}$ and $C S$. Numbers in parentheses indicate the number of repetitions.

transgene. The dunce gene product has been shown to be required in somatic and germ cells of females for normal fertility (Bellen and Kiger, 1988). Since the time when dunce gene activity is required for fertility was unknown, we exposed the females to cycling heat shock conditions starting shortly after eclosion. Homozygous $d n c$ females, carrying two copies of a hspdnc transposon (hspdnc26-dnc $c^{\mathrm{ML}}$ or hspdnc54-dnc ${ }^{\mathrm{ML}}$ ) were kept uIIder these conditions for four days and then mated. The $d n c^{\mathrm{ML}}$ females without the transposon and the parental $y w f^{36 a}$ strain were treated identically. Heat treatment did not affect the fertility of $y w f^{36 a}$ control flies (Table 1). More than $80 \%$ of the females produced progeny and in equal numbers whether exposed to cycling conditions or not (21 progeny on average for the $1.5 \mathrm{~d}$ laying period). The $d n c^{\mathrm{ml}}$ flies were completely sterile, with or without heat treatment. In contrast, about half of the $d n c^{\mathrm{ML}} \mathrm{fe}-$ males transformed with either hspdnc 26 or hspdnc54 were fertile, producing about four progeny on average. Fertility was restored whether the mating was performed at room temperature or under cycling conditions (Table 1). Transformed $d n c^{\mathrm{ML}}$ females were sterile without heat shock except for two hspdnc54 females. Fertility of these females could be due to basal expression from the hsp70 promoter or to the very low level of fertility previously described for the $d n c^{\mathrm{ML}}$ allele (Salz et al., 1982).

In summary, heat-induced expression from hspdnc transgenes leads to partial rescue of the sterility of $d n c^{\mathrm{ML}}$ females. Induced expression is required in the female parent prior to mating, since rescue was not observed when induction was simultaneous with the mating/egg laying period.

\section{Discussion}

We have generated transgenic $d n c$ flies containing transposons which provide heat shock-induced expression of either a Dro- sophila or a rat cAMP PDE. The transposons were used to determine whether induction of the gene product just prior to training during adulthood can lead to behavioral rescue. The rat and the Drosophila dunce minigenes were both capable of improving the $d n c$ learning deficit when induced shortly before training in an olfactory classical conditioning paradigm. This was observed with two different alleles, with several different transposons and in different genetic backgrounds. Heat-induced expression of the transposons in adult $d n c$ females partially rescued the $d n c$ sterility phenotype as well. These findings demonstrate that expression of dunce activity during adulthood is critical for normal learning and normal fertility, therefore implying a physiological role for the gene product.

\section{Requirement for dunce activity in learning}

The learning indices of two different $d n c$ mutants, $d n c^{\mathrm{ML}}$ and $d n c^{1}$, were significantly ameliorated after induction of the PDE transgenes with heat shock. The improvement was more pronounced for transgenic $d n c^{\mathrm{ML}}$ flies than for transgenic $d n c^{\prime}$ flies. This suggests that the nature of the mutation in the given mutant allele affects the degree of rescue. Indeed, learning was not significantly improved when the hspRD1-12 transposon was introduced into the $d n c$ null allele, $d n c^{\mathrm{M} / 4}$ (data not shown). Perhaps the requirement for dunce activity in the learning process is quantitative, with extreme alleles being recalcitrant to rescue at the level of activity supplied by a heat-shock driven transgene. Other dunce phenotypes may require a different threshold of activity. Already mated wild-type females, for example, reject courting males (Kubli, 1992). The sex-peptide, a peptide transferred during copulation from the male to the female, induces the same response when injected into virgin females (Chen et 
Table 1. Rescue of the $d n c$ female sterility by hspdnc transposons

\begin{tabular}{|c|c|c|c|c|}
\hline \multirow[b]{2}{*}{ Female genotype } & \multicolumn{2}{|c|}{ o hs (cycling) $4 \mathrm{~d}$ w/o males $(a)$} & \multicolumn{2}{|c|}{ \& RT $4 \mathrm{~d}$ w/o males $(b)$} \\
\hline & $+\delta$, hs $1.5 \mathrm{~d}(c)$ & $+\delta$, RT $1.5 \mathrm{~d}(d)$ & $+\delta$, hs $1.5 \mathrm{~d}(e)$ & $+\delta$, RT $1.5 \mathrm{~d}(f)$ \\
\hline$y w f^{f r a}$ & $87 \%(27 / 31)$ & $83 \%(25 / 30)$ & $85 \%(17 / 20)$ & $82 \%(23 / 28)$ \\
\hline & $19.5 \pm 1.9$ & $19.2 \pm 1.5$ & $23 \pm 3.2$ & $20.1 \pm 3.5$ \\
\hline $\mathrm{dnc}^{\mathrm{MI}}$ & $0 \%(0 / 14)$ & $0 \%(0 / 17)$ & $0 \%(0 / 9)$ & $0 \%(0 / 12)$ \\
\hline hspdnc26-dnc $c^{\mathrm{ML}}$ & $\begin{array}{c}57.8 \%(48 / 83) \\
3.1 \pm 0.3\end{array}$ & $\begin{array}{c}48 \%(38 / 80) \\
3.3 \pm 0.4\end{array}$ & $0 \%(0 / 16)$ & $0 \%(0 / 17)$ \\
\hline hspdnc54-dnc $c^{\mathrm{ML}}$ & $\begin{array}{c}45 \%(21 / 47) \\
4.9 \pm 1.1\end{array}$ & $\begin{array}{c}35.7 \%(15 / 42) \\
4.8 \pm 0.2\end{array}$ & $0 \%(0 / 39)$ & $\begin{array}{l}5.4 \%(2 / 37) \\
4.0\end{array}$ \\
\hline
\end{tabular}

Virgin females were kept in an incubator cycling between $37^{\circ} \mathrm{C}$ (hs) and $25^{\circ} \mathrm{C}$ for $1 \mathrm{hr}$ and $2 \mathrm{hr}$, respectively $(a)$ or at room temperature (RT) for $4 \mathrm{~d}$. (b). They were then individually mated to three Canton-S males either under cycling conditions (hs) as above ( $c$ and $e$ ) or at room terperature (RT) ( $d$ and $f$ ). The parents were removed after $1.5 \mathrm{~d}$ and all of the crosses kept at room temperature. The number of progeny produced was counted $20 \mathrm{~d}$ after mating. The percentage of females producing offspring (absolute numbers are in parentheses) and the average number of progeny \pm SEM per fertile female is shown. The strains used were $y w d n c^{M /} f^{i s i}\left(d n c^{m t}\right)$, the parental line $y w$

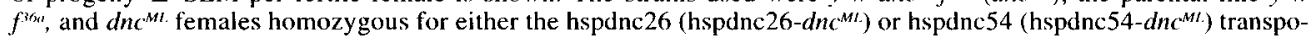
sons.

al., 1988; Aigaki et al., 1991). The change in receptivity due to the sex-peptide is absent in dunce females (Chapmann et al., unpublished observations), but restored partially when the rat transgene is induced in the $d n c^{\mathrm{M} / 4}$ mutant (I. Fleischmann and E. Kubli, personal communication).

Although a threshold requirement can explain the failure to rescue learning in $d n c^{\mathrm{M} 14}$, it is inconsistent with the difference between $d n c^{\prime}$ and $d n c^{\mathrm{ML}}$. The $d n c^{\mathrm{ML}}$ mutation is an extreme hypomorphic allele with about $10 \%$ of the normal dunce PDE activity whereas $d n c^{1}$ retains more than $50 \%$ of the activity (Davis and Kiger, 1981). However, the $d n c^{\prime}$ encoded enzyme must be structurally altered since it is known to be more thermolabile (Kauvar, 1982). Conceivably, the $d n c^{\prime}$ enzyme could compete with the induced enzyme in some unknown biochemical or cellular function, thereby reducing the effectiveness of the transgenically supplied enzyme for rescue of the learning deficit. Consistent with this interpretation, lower learning scores have been observed in $d n c^{\prime}$ homozygote mutants (possessing more than $50 \%$ of wild-type PDE activity) than in null alleles over a wild-type chromosome with comparable PDE activity levels (Tully and Gold, 1993).

The complexity of the dunce gene with its more than 10 transcripts might provide an alternative explanation for different rescue levels and/or incomplete rescue. The different dunce mutations may affect different subsets of dunce products which differ in localization, stability, or modifications. The function of some of these may be rescued more efficiently than others by the cDNA expressed by the transgene.

Alternatively, rescue of the hypomorphic alleles, but not the null allele might reflect a requirement for the gene during development to establish structures or functions that participate in adult learning processes. The levels of endogenous activity in the two hypomorphs and/or the uninduced activity from the transgenically supplied enzyme might be sufficient to provide for this putative developmental function, but insufficient in the null allele. Induction of the enzyme in a null allele background at various times during development might distinguish these alternatives more clearly. Unfortunately, the demanding nature of the behavioral experiments makes this endeavor impractical.

PDE activity in some transgenic $d n c$ flies reaches wild-type levels after heat shock when measured in whole fly homogenates. However, since the heat shock promoter confers expres- sion in most cells, the induced levels in cell types thought to be critical for learning such as these of the mushroom bodies (Nighorn et al., 1991; Qiu and Davis, 1993; de Belle and Heisenberg, 1994), must be substantially lower than in wild type. Nevertheless we observed partial rescue in the transformed flies. This can be explained by the findings of Qiu and Davis (1993) that certain mutants with markedly reduced dunce PDE levels in mushroom bodies but with an otherwise normal expression in other brain neuropil, show normal learning immediately after training but have a reduced memory. The high levels of dunce PDE found in mushroom bodies have therefore been suggested to be primarily required for memory processes, with low levels in mushroom bodies or in other brain neuropil required for initial learning (Qiu and Davis, 1993). A low threshold requirement for initial learning can also explain the partial rescue observed in some transformed lines in the absence of heat shock, due to the leakiness of the hsp promoter. However, complete rescue seems to require substantially higher PDE levels than those induced in the relevant cells after heat shock. This would explain why we were not able to obtain complete rescue after heat induction of the enzyme.

Alternatively, complete rescue might require some developmental PDE function not provided in the transformed flies. The partial rescue might therefore reflect the double requirement of the dunce PDE for processes in development and during learning.

Our data demonstrate a major physiological requirement for the dunce PDE in the learning/memory process. At present, little is known about the specific cellular mechanisms by which cAMP regulates learning in the adult fly. Given the rapidity of rescue after heat shock it seems likely that physiological aspects of neuronal plasticity are disrupted by the $d n c$ mutation and that the rescue was achieved by reversing this disruption. This might happen through the activation of PKA, or by direct effects of cAMP on other proteins such as a cAMP activated $\mathrm{K}^{+}$channel that is persistently activated in anc larval neuromuscular junctions (Delgado et al., 1991). Drain et al. (1991) and Skoulakis et al. (1993) have demonstrated that a major mediator of cAMP signaling involved in learning and memory is PKA. Zhong et al. (1991) demonstrated a lack of facilitation or potentiation at the neuromuscular junction in $d n c$ mutants under conditions evoking these responses in normal larvae. It is conceivable that 
the plasticity of synapses in the adult brain might be disrupted in $d n c$ mutants in a similar way with rescue occurring by restoring this plasticity.

\section{Requirement of the dunce PDE for female fertility}

The $d n c$ homozygous females are sterile. Mosaic analysis has revealed a requirement for the protein in somatic cells for egglaying and in gern cells for proper embryonic development (Bellen et al., 1987). The time and specific location of the requirement in somatic cells has not been elucidated. Staining of male and female flies with an anti-dunce antibody revealed sexually dimorphic staining of the abdominal ganglion, with females showing more intense staining than males (Nighorn et al., 1991). This might point to abdominal ganglion involvement in the female specific action of dunce since the ovarian musculature is innervated by this ganglion (Miller, 1950). Subjecting newly eclosed $d n c$ females containing a hspdnc transposon to cycling heat shock conditions for four days conferred partial fertility, demonstrating rescue of both the somatic and the germline-specific defects. The partial rescue could be due to insufficient enzyme levels or the possibility that PDE was not provided at all appropriate stages. Remarkably, the level of rescue was the same whether the subsequent mating and egg laying was performed with or without heat shock. In the time interval covered by the heat treatment, oocytes mature in virgin females and many maternal RNAs and proteins are made in the nurse cells and delivered to the oocyte (King, 1970). Part of the germline requirement for dunce is the establishment of appropriate cAMP levels and distribution in unfertilized eggs (Whitehouse-Hills et al., 1992). This was also suggested by the lethality caused by PDE expression from our injected transformation constructs.

\section{A rat homolog of dunce rescues the learning deficit}

A remarkable observation made here is that a transgene carrying a rat homolog of dunce can conditionally improve the learning deficits of $d n c$ mutants. This indicates functional conservation between the Drosophila and mammalian "dunce" homologue that allows the rat enzyme to substitute for the Drosophila enzyme in a complex process such as learning. The rat and Drosophila $\mathrm{c} \Lambda \mathrm{MP}$ PDEs are highly homologous and it seems likely that a key feature for their functional equivalence lies in their very similar kinetic properties. Both have been shown to code for low $K_{m}$, cAMP-specific PDEs (Henkel-Tigges and Davis, 1989). However, other properties for normal function, such as subcellular distribution and biological regulation, must also be conserved sufficiently to allow the rat homolog to fill the role of the fly gene.

\section{References}

Aigaki T, Fleischmann I, Chen PS, Kubli E (1991) Ectopic expression of sex peptide alters reproductive behavior of female $D$. melanogaster. Neuron 7:557-563.

Alkon DL, Nelson TJ (1990) Specificity of molecular changes in neurons involved in memory storage. FASEB J 4:1567-1576.

Balling A, Technau GM, Heisenberg M (1987) Are the structural changes in adult Drosophila mushroom bodies memory traces? Studies on biochemical learning mutants. J Neurogenet 4:65-73.

Beavo JA, Reifsnyder DH (1990) Primary sequence of cyclic nucleotide phosphodiesterase isozymes and the design of selective inhibitors. Trends Pharmacol Sci 11:150-155.

Bellen HJ, Kiger JA (1988) Maternal effects of general and regional specificity on embryos of Drosophila melanogaster caused by dunce and rutahaga mutant combinations. Rouxs Arch Dev Biol 197:258268.

Bellen HJ, Gregory BK, Olsson CL, Kiger JA (1987) Two Drosophila learning mutants, dunce and rutabaga, provide evidence of a maternal role for cAMP on embryogenesis. Dev Biol 121:432-444.

Bishop JG, Corces VG (1988) Expression of an activated ras gene causes developmental abnormalities in transgenic Drosophila melanogaster. Genes Dev 2:567-577.

Boggs RT, Gregor P, Idriss S, Belote JM, McKeown M (1987) Regulation of sexual differentiation in $D$. melanogaster via alternative splicing of RNA from the transformer gene. Cell 50:739-747.

Bonner JJ, Pardue ML (1976) The effect of heat shock on RNA synthesis in Drosophila tissues. Cell 8:43-50.

Bonner JJ, Parks C, Parker-Thornburg J, Mortin MA, Pelham HRB (1984) The use of promoter fusions in Drosophila genetics: isolation of mutations affecting the heat shock response. Cell 37:979-991.

Byers D, Davis RL, Kiger JA Jr (1981) Defect in cyclic AMP phosphodicstcrase duc to the dunce mutation of lcarning in Drosophila melanogaster. Nature 289:79-81.

Byrne JH (1987) Cellular analysis of associative learning. Physiol Rev $67: 329-439$.

Chen CN, Denome S, Davis RL (1986) Molecular analysis of cDNA clones and the corresponding genomic coding region of the Drosophila dunce $e^{+}$locus, the structure gene for cAMP phosphodiesterase. Proc Natl Acad Sci USA 86:3599-3603.

Chen PS, Stumm-Zollinger E, Aigaki T, Balmer J, Bienz M, Böhlen P (1988) A male accessory gland peptide that regulates reproductive behavior of female D. melanogaster. Cell 54:291-298.

Corfas G, Dudai Y (1991) Morphology of a sensory neuron in Dro sophila is abnormal in memory mutants and changes during aging. Proc Natl Acad Sci USA 88:7252-7256.

Crow T (1988) Cellular and molecular analysis of associative learning and memory in Hermissenda. Trends Neurosci 11:136-142.

Davis R (1988) Mutational analysis of Drosophila phosphodiesterases. Methods Enzymol 159:786-792.

Davis RL (1993) Mushroom bodies and Drosophila learning. Neuron 11:1-14

Davis RL, Kiger JA Jr (1981) dunce mutants of Drosophila melanogaster: mutants defective in the cyclic AMP phosphodiesterase enzyme system. J Cell Biol 90:101-107.

Davis RL, Takayasu H, Eberwine M, Myres J (1989) Cloning and characterization of mammalian homologs of the Drosophila dunce ${ }^{+}$ gene. Proc Natl Acad Sci USA 86:3604-3608.

de Belle JS, Heisenberg M (1994) Associative odor learning in Drosophila abolished by chemical ablation of mushroom bodies. Science 263:692-695.

Delgado R, Hidalgo P, Diaz F, Latorre R, Labarca P (1991) A cyclic AMP-activated $\mathrm{K}^{+}$channel in Drosophila larval muscle is persistently activated in dunce. Proc Natl Acad Sci USA 88:557-560.

Drain P, Folkers E, Quinn WG (1991) cAMP-dependent protein kinase and the disruption of learning in transgenic flies. Neuron 6:71-82.

Dudai Y (1977) Properties of learning and memory in Drosophila melanogaster. J Comp Physiol 114:69-89.

Dudai Y (1983) Mutations affect storage and use of memory differentially in Drosophila. Proc Natl Acad Sci USA 80:5445-5448.

Dudai Y, Jan Y-N, Byers D, Quinn W, Benzer S (1976) dunce: a mutant of Drosophila deficient in learning. Proc Natl Acad Sci USA 73: $1684-1688$.

Dudai Y, Buxbaum J, Corfas G, Orgad S, Segal D, Sher B, Uzzan A, Zvi S (1986) Defective cAMP metabolism and defective memory in Drosophila. Acta Biochim Biophys Hung 21:177-192.

Duerr JS, Quinn WG (1982) Three Drosophila mutations that block associative learning also affect habituation and sensitization. Proc Natl Acad Sci USA 79:3646-3650.

Dura JM, Preat T, Tully T (1993) Identification of linotte: a new gene affecting learning and memory in Drosophila melanogaster. $\mathrm{J}$ Neurogenet $9: 1-14$.

Erber J, Masuhr T, Menzel R (1980) Localization of short-term memory in the brain of the bee, Apis mellifera. Physiol Entomol 5:343358.

Ewer J, Hamblen-Coyle M, Rosbash M, Hall JC (1990) Requirement for period gene expression in the adult and not during development for locomotor activity rhythms of imaginal Drosophila melanogaster. J Neurogenet 7:31-73.

Folkers E, Spatz HCh (1984) Visual learning performance of Drosophila melanogaster is altered by neuropharmaca affecting phosphodiesterase activity and acetylcholine transmission. $J$ Insect Physiol 30:957-965. 
Gailey D $\Lambda$, Villella $\Lambda$, Tully $T$ (1991) Reassessment of the effect of biological rhythm mutations on learning in Drosophila melanogaster. J Comp Physiol A 169:685-697.

Grant SGN, O’Dell TJ, Karl KA, Stein PL, Soriano P, Kandel ER (1992) Impaired long-term potentiation, spatial learning, and hippocampal development in fyn mutant mice. Science 258:1903-1910.

Han PL, Levin LR, Reed RR, Davis RL (1992) Preferred expression of the Drosophila rutabaga gene in mushroom bodies, neural centres for learning in insects. Neuron 9:619-627.

Heisenberg M, Borst A, Wagner S, Byers D (1985) Drosophila mushroom body mutants are deficient in olfactory learning. J Neurogenet $2: 1-30$.

Henkel-Tigges J, Davis RL (1990) Rat homologs of the Drosophila dunce gene code for cyclic AMP phosphodiesterases sensitive to rolipram and RO20-1724. Mol Pharmacol 37:7-10.

Kandel ER, Schacher S, Castellucci VF, Goelet P (1987) The long and short of memory in Aplysia: a molecular perspective. In: Fidia Research Foundation Neuroscience Award Lectures 1986, pp 7-47. Padua: Liviana.

Kauvar LM (1982) Defective cyclic adenosine 3':5'-monophosphate phosphodiesterase in the Drosophila memory mutant dunce. J Neurosei $2: 1347-1358$.

King RC (1970) Ovarian development in Drosophila melanogaster. New York: Academic.

Kubli E (1992) My favorite molecule: the sex-peptide. Bioassays 7:779-784.

Levin LR, Han PL, Hwang PM, Feinstein PG, Davis RL, Reed RR (1992) The Drosophila learning and memory gene, rutabaga, encodes a $\mathrm{Ca}^{2+} /$ calmodulin-responsive adenylyl cyclase. Cell 68:479489.

Lis JT, Simon JA, Sutton CA (1983) New heat shock puffs and $\beta$-galactosidase activity resulting from transformation of Drosophila with an hsp70-lacZ hybrid gene. Cell 35:403-410.

Livingstone MS, Sziber PP, Quinn WG (1984) Loss of calcium/calmodulin responsiveness in adenylate cyclase of rutabaga: a Drosophila learning mutant. Cell 37:205-215.

Malinow R, Madison DV, Tsien RW (1988) Persistent protein kinase activity underlying long-term potentiation. Nature 335:820-824.

Miller A (1950) The internal anatomy and histology of the imago of Drosophila melanogaster. In: Biology of Drosophila (Demerec M, ed), pp 420-531. New York: Wiley.

Nighom A, Healy MJ, Davis RL (1991) The cyclic AMP phosphodiesterase encoded by the Drosophila dunce gene is concentrated in the mushroom body neuropil. Neuron 6:455-467.

Petersen RB, Lindquist S (1989) Regulation of hsp70 synthesis by messenger RNA degradation. Cell Regul 1:135-149.

Pirrotta V (1988) In: A survey of molecular cloning vectors and their uses (Rodriguez RL, Denhardt DT, eds), pp 427-445. Boston: Butterworths.
Qiu Y, Davis RL (1993) Genetic dissection of the learning/memory gene dunce of Drosophila melanogaster. Genes Dev 7:1447-1458.

Qiu Y, Chan CN, Malone T, Richter L, Beckendorf SK, Davis RL (1991) Characterization of the memory gene dunce of Drosophila melanogaster. J Mol Biol 222:553-565.

Quinn WG, Harris WA, Benzer S (1974) Conditioned behavior in Drosophila melanogaster. Proc Natl Acad Sci USA 71:707-712.

Salz HK, Davis RL, Kiger JA (1982) Genetic analysis of chromosome 3D4 in Drosophila melanogaster: the dunce and sperm-amotile genes. Genetics 100:587-596.

Schacher S, Castellucci VF, Kandel ER (1988) cAMP evokes longterm facilitation in Aplysia sensory neurons that requires new protein synthesis. Science 240:1667-1669.

Schildberger K (1984) Multimodal interneurons in the cricket brain: properties of identified extrinsic mushroom body cells. J Comp Physiol A 154:71-79.

Scholz KP, Byrne JH (1988) Intracellular injection of cAMP induces a long-term reduction of neuronal $\mathrm{K}^{+}$currents. Science 240:16641666.

Silva AJ, Paylor R, Wehner JM, Tonegawa S (1992) Impaired spatial learning in $\alpha$-calcium-calmodulin kinase II mutant mice. Science 257 : 206-211.

Skoulakis EMC, Kalderon D, Davis RL (1993) Preferential expression of the catalytic subunit of PKA in mushroom bodies and its role in learning and memory. Neuron 11:197-208.

Spradling AC, Rubin GM (1982) Transposition of cloned P elements into Drosophila germ line chromosomes. Science 218:341-347.

Steller H, Pirrotta V (1984) Regulated expression of genes injected into early Drosophila embryos. EMBO J 3:165-173.

Steller H, Pirrotta V (1985) Expression of the Drosophila white gene under the control of the hsp70 promoter. EMBO J 4:3765-3772.

Tempel BL, Bonini N, Dawson DR, Quinn WG (1983) Reward learning in normal and mutant Drosophila. Proc Natl Acad Sci USA 80: $1482-1486$.

Tomlinson A, Kimmel BE, Rubin GM (1988) rough, a Drosophila homeobox gene required in photoreceptors R2 and R5 for inductive interactions in the developing eye. Cell 56:771-784.

Tully T, Gold D (1993) Differential effects of dunce mutations on associative learning and memory in Drosophila. J Neurogenet 9:5571.

Tully T, Quinn WG (1985) Classical conditioning and retention in normal and mutant Drosophila melanogaster. J Comp Physiol A 157: 263-277.

Whitehouse-Hills S, Bellen HI, Kiger JA (1992) Embryonic cAMP and developmental potential in Drosophila melanogaster. Rouxs Arch Dev Biol 201:257-264.

Zhong Y, Wu C-F (1991) Altered synaptic plasticity in Drosophila memory mutants with a defective cyclic AMP cascade. Science 251: 198-201.

Zhong Y, Budnik V, Wu C-F (1992) Synaptic plasticity in Drosophila memory and hyperexcitable mutants: role of cAMP cascade. J Neuroscience 12:644-651. 